\title{
PATCHING AND ANALYTIC ISOMORPHISMS
}

\author{
STEVEN E. LANDSBURG
}

(Communicated by Louis J. Ratliff, Jr.)

Abstract. We give a particularly simple proof of the Milnor Patching Property for a class of diagrams that includes analytic isomorphism squares.

Consider a commutative diagram of commutative rings

(1)

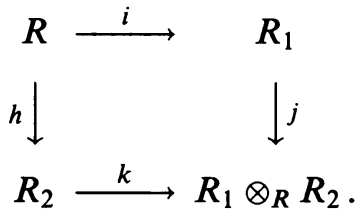

A collection of patching data is a triple $\left(P_{1}, P_{2}, \phi\right)$, where $P_{i}$ is a finitely generated projective $R_{i}$-module and

$$
\phi: P_{1} \otimes_{R} R_{2} \stackrel{\approx}{\rightarrow} R_{1} \otimes_{R} P_{2}
$$

is an isomorphism of $\left(R_{1} \otimes_{R} R_{2}\right)$-modules.

The category of finitely generated projective $R$-modules maps functorially to the category of patching data via

$$
P \mapsto\left(P \otimes_{R} R_{1}, R_{2} \otimes_{R} P, \phi\right)
$$

with $\phi$ the obvious identification $p \otimes 1 \otimes 1 \mapsto 1 \otimes 1 \otimes p$.

We say that (1) is a Milnor patching diagram if (2) is an equivalence of categories, with an inverse equivalence given by

$$
\left(P_{1}, P_{2}, \phi\right) \mapsto\left\{\left(p_{1}, p_{2}\right) \mid p_{i} \in P_{i} \text { and } \phi\left(p_{1} \otimes 1\right)=1 \otimes p_{2}\right\} \text {. }
$$

In [M, Chapter 2], Milnor showed that (1) is a Milnor patching diagram if it is a pullback in the category of rings and $j$ is onto.

Another class of Milnor patching diagrams arises in the following way.

Analytic Isomorphism Theorem. Let

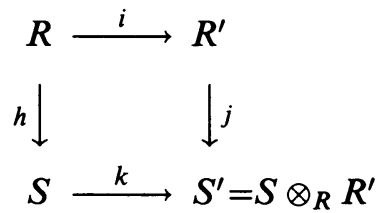

Received by the editors September 24, 1990.

1980 Mathematics Subject Classification (1985 Revision). Primary 13C10. 
be a pullback square of commutative rings. Suppose that there exists a multiplicatively closed subset $\Sigma \subset R$ such that:

(i) $R^{\prime}=\Sigma^{-1} R$.

(ii) For every $f \in \Sigma$, the induced map $R / f R \rightarrow S / f S$ is an isomorphism.

Then (3) is a Milnor patching diagram.

(Hypothesis (ii) is sometimes expressed by saying that $h$ is an analytic isomorphism along $\Sigma$.)

The analytic isomorphism theorem was proved by Landsburg in [La] and (implicitly) by Lindel in [Li] (cf. the proof of Lemma 4 in [Li]). [La] requires an additional generic flatness hypothesis and [Li] requires an additional noetherian hypothesis, but neither of these is necessary, as we shall see below.

Notice that if $\Sigma$ consists of elements that are nonzero-divisors on both $R$ and $S$, then the injectivity of the induced maps in (ii) becomes equivalent to the pullback property which is assumed earlier in the statement of the theorem.

Here we shall generalize the analytic isomorphism theorem in two directions, by weakening the hypothesis and strengthening the conclusion. The resulting proof of the analytic isomorphism theorem itself is undoubtedly the ultimate in simplicity.

To state the new, stronger conclusion, say that (1) is a strong Milnor patching diagram if it is a pullback in the category of commutative rings, and also:

For every $n \geq 1$, and every $u \in G L_{n}\left(R_{1} \otimes_{R} R_{2}\right)$,

$$
\left(\begin{array}{cc}
u & 0 \\
0 & u^{-1}
\end{array}\right) \in j\left(G L_{2 n}\left(R_{1}\right)\right) \cdot k\left(G L_{2 n}\left(R_{2}\right)\right) \subset G L_{2 n}\left(R_{1} \otimes_{R} R_{2}\right) .
$$

The methods of $[\mathrm{M}]$ demonstrate that strong Milnor patching implies Milnor patching. We conjecture that the converse is true. (This conjecture is based on a variety of partial classification theorems for Milnor patching diagrams, some not yet published.) However, strong Milnor patching conveys significantly more information. For example, it easily implies that if $P$ is a finitely generated projective $R$-module of rank $n$ which becomes free over each $R_{i}$, then $P$ can be generated by $2 n$ elements.

Now we can state and prove our main theorem.

Generalized Analytic Isomorphism Theorem. Let

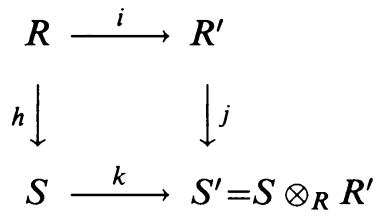

be a pullback square of commutative rings. Suppose that there exists a multiplicatively closed subset $\Sigma \subset R$ with the following properties:

(i) For every $r^{\prime} \in R^{\prime}$, there exists an element $f \in \Sigma$ such that $f r^{\prime} \in i(R)$.

(ii) For every $f \in \Sigma$, let $I_{f}=i^{-1}\left(f R^{\prime}\right) \subset R$ and let $K_{f}=k^{-1}\left(f S^{\prime}\right) \subset S$. Then the map induced by $h, I_{f} / f I_{f} \rightarrow K_{f} / f K_{f}$, is an isomorphism.

(iii) No element of $\Sigma$ is a zero-divisor on $R^{\prime}$ or $S^{\prime}$.

Then (4) is a strong Milnor patching diagram.

Note that condition (iii) does not require $\Sigma$ to consist of nonzero-divisors on $R$ and $S$. 
If we take $R^{\prime}=\Sigma^{-1} R$, this is a strengthening of the Analytic Isomorphism Theorem. If we take $\Sigma=\{1\}$ we recover Milnor's Theorem.

Proof. Pick $u \in G L_{n}\left(S^{\prime}\right)$. By (i), there exist $f \in \Sigma$ and $\alpha, \beta \in M_{n}(S)$ such that $k(\alpha)=f u$ and $k(\beta)=f u^{-1}$. Write $I$ for the $n \times n$ identity matrix. Then $f^{2} I-\alpha \beta \in M_{n}(S)$ maps to zero in $M_{n}\left(S^{\prime}\right)$ and consequently (by the pullback property) lifts to a matrix $x \in \operatorname{ker}\left(M_{n}(R) \rightarrow M_{n}\left(R^{\prime}\right)\right)$.

By repeated use of (ii), we can write $\alpha=h\left(r_{1}\right)+f^{4} s_{1}$ and $\beta=h\left(r_{2}\right)+f^{4} s_{2}$, with $r_{\nu} \in M_{n}\left(I_{f}\right) \subset M_{n}(R)$ and $s_{\nu} \in M_{n}\left(K_{f}\right) \subset M_{n}(S)$. Write $i\left(r_{\nu}\right)=f a_{\nu}$.

Now $h\left(f^{2} I-r_{1} r_{2}-x\right) \in f^{4} S \subset M_{n}\left(f^{2} K_{f^{2}}\right)$, so by condition (ii) applied to $f^{2}$, we have $f^{2} I-r_{1} r_{2}-x \in M_{n}\left(f^{2} I_{f^{2}}\right)$. Consequently, $f^{2}\left(I-a_{1} a_{2}\right)=$ $i\left(f^{2}-r_{1} r_{2}-x\right) \in f^{4} M_{n}\left(R^{\prime}\right) \subset M_{n}\left(f^{3} R^{\prime}\right)$. Write $f^{2}\left(I-a_{1} a_{2}\right)=f^{3} a^{\prime}$. By (iii), we have $I-a_{1} a_{2}=f a^{\prime}$.

In a similar manner construct $a^{\prime \prime} \in M_{n}\left(R^{\prime}\right)$ such that $I-a_{2} a_{1}=f a^{\prime \prime}$.

Now let

$$
r^{\prime}=\left(\begin{array}{cc}
a^{\prime} & a_{1} \\
-a_{2} & f I
\end{array}\right) \in M_{n}\left(R^{\prime}\right)
$$

and note that $r^{\prime}$ is invertible because its (two-sided) inverse is given by

$$
\left(r^{\prime}\right)^{-1}=\left(\begin{array}{cc}
f I & -a_{1} \\
a_{2} & a^{\prime \prime}
\end{array}\right) \text {. }
$$

Let

$$
s=\left(\begin{array}{cc}
\alpha & -I+\alpha \beta s_{1} \beta \\
I-f^{2} s_{2} \alpha & \beta s_{1} \beta+f^{2} s_{2}-f^{2} s_{2} \alpha \beta s_{1} \beta
\end{array}\right) \in M_{n}(S)
$$

and note that $s$ is invertible because its (two-sided) inverse is given by

$$
s^{-1}=\left(\begin{array}{cc}
\beta s_{1} \beta+f^{2} s_{2}-f^{2} \beta s_{1} \beta \alpha s_{2} & I-\beta s_{1} \beta \alpha \\
-I+f^{2} \alpha s_{2} & \alpha
\end{array}\right) .
$$

Finally, one checks easily that

$$
j\left(r^{\prime}\right) \cdot k(s)=\left(\begin{array}{cc}
u & 0 \\
0 & u^{-1}
\end{array}\right) .
$$

(This equality is most easily verified after first multiplying each side by $f^{2}$, which is allowable by (iii).)

Remark. We have not used the full strength of the injectivity of $I_{f} / f I_{f} \rightarrow$ $K_{f} / f K_{f}$. The only consequence of this that we need is the following: for every $f \in \Sigma$ and $r \in R$, if $i(r) \in f^{2} R^{\prime}$ and $h(r) \in f^{4} S$, then $i(r) \in f^{3} R^{\prime}$.

\section{REFERENCES}

[La] S. Landsburg, Patching theorems for projective modules, J. Pure Appl. Algebra 22 (1981), 261-277.

[Li] H. Lindel, Projektive Moduln Über Polynomringen $A\left[T_{1}, \ldots, T_{m}\right]$ mit Einem Regularen Grundring $A$, Manuscripta Math. 23 (1978), 143-154.

[M] J. Milnor, An introduction to algebraic $K$-theory, Princeton Univ. Press, Princeton, NJ, 1971.

Department of Mathematics, Colorado State University, Fort Collins, Colorado 